\title{
Evaluation of predictive models for delayed graft function of deceased kidney transplantation
}

\author{
Huanxi Zhang ${ }^{1, *}$, Linli Zheng ${ }^{2, *}$, Shuhang Qin ${ }^{2, *}$, Longshan Liu ${ }^{1}$, Xiaopeng Yuan ${ }^{1}$, \\ Qian $\mathrm{Fu}^{1}$, Jun $\mathrm{Li}^{1}$, Ronghai Deng ${ }^{1}$, Suxiong Deng ${ }^{1}$, Fangchao $\mathrm{Yu}^{1}$, Xiaoshun $\mathrm{He}^{1,3}$ \\ and Changxi Wang ${ }^{1,3}$ \\ ${ }^{1}$ Organ Transplant Center, The First Affiliated Hospital, Sun Yat-Sen University, Guangzhou 510080, China \\ ${ }^{2}$ Zhongshan School of Medicine, Sun Yat-Sen University, Guangzhou 510080, China \\ ${ }^{3}$ Guangdong Provincial Key Laboratory on Organ Donation and Transplant Immunology, Guangzhou 510080, China \\ *These authors contributed equally to this work
}

Correspondence to: Changxi Wang, email: wangchx@mail.sysu.edu.cn

Xiaoshun He, email: gdtrc@163.com

Keywords: delayed graft function; graft survival; deceased kidney transplantation; prediction models

Received: September 16, $2017 \quad$ Accepted: October 27, $2017 \quad$ Published: November 27, 2017

Copyright: Zhang et al. This is an open-access article distributed under the terms of the Creative Commons Attribution License 3.0 (CC BY 3.0), which permits unrestricted use, distribution, and reproduction in any medium, provided the original author and source are credited.

\section{ABSTRACT}

Background: This study aimed to evaluate the predictive power of five available delayed graft function (DGF)-prediction models for kidney transplants in the Chinese population.

Results: Among the five models, the Irish 2010 model scored the best in performance for the Chinese population. Irish $\mathbf{2 0 1 0}$ model had an area under the receiver operating characteristic (ROC) curve of $\mathbf{0 . 7 3 7}$. Hosmer-Lemeshow goodnessof-fit test showed that the Irish $\mathbf{2 0 1 0}$ model had a strong correlation between the calculated DGF risk and the observed DGF incidence $(p=0.887)$. When Irish 2010 model was used in the clinic, the optimal upper cut-off was set to 0.5 with the best positive likelihood ratio, while the lower cut-off was set to 0.1 with the best negative likelihood ratio. In the subgroup of donor aged $\leq 5$, the observed DGF incidence was significantly higher than the calculated DGF risk by Irish 2010 model ( $27 \%$ vs. 9\%).

Materials and Methods: A total of $\mathbf{7 1 1}$ renal transplant cases using deceased donors from China Donation after Citizen's Death Program at our center between February 2007 and August 2016 were included in the analysis using the five predictive models (Irish 2010, Irish 2003, Chaphal 2014, Zaza 2015, Jeldres 2009).

Conclusions: Irish $\mathbf{2 0 1 0}$ model has the best predictive power for DGF risk in Chinese population among the five models. However, it may not be suitable for allograft recipients whose donor aged $\leq 5$-year-old.

\section{INTRODUCTION}

Kidney transplantation has become a routine procedure in the treatment of patients with irreversible kidney failure [1]. Delayed graft function (DGF) is a complication after kidney transplantation and is a manifestation of acute kidney injury commonly defined as requiring dialysis within the first week post-transplantation [2]. The reported incidence of DGF in deceased donors has increased over the past decades [3], and is expected to rise further due to the use of expanded criteria donors (ECD) and donation after cardiac death (DCD) who show higher DGF rate than donation after brain death (DBD) [4-6]. Over the last three decades, the reported incidences of DGF in adult recipients from deceased donor kidney transplantation range from 15 to $30 \%$ [7].

DGF is a major obstacle for successful transplantation as it causes post-transplantation oliguria, increased allograft immunogenicity and risk of acute rejection episodes, and decreased long-term survival [8]. Due to the adverse influences of DGF on the transplantation outcome, significant efforts have been made to identify risk factors for DGF [7, 9-11]. Identification of risk factors for DGF is helpful for early preventive management. In addition, in 
attempting to develop an easy method to predict DGF, several clinical algorithms have been proposed and are reported to have good predictive power for DGF [12-16]. In 2003, Irish et al. have developed a multivariable logistic regression model including 16 variables of donors and recipients at the time of transplantation to predict the likelihood of DGF occurrence using the data on 13,846 cases of deceased donor kidney transplantation from the United States Renal Data System registry [14]. In 2010, Irish et al. further refined their predictive model to include warm ischemia time (WIT, total 17 variables) using the data from 24,337 cases of deceased donor kidney transplantation, which has a diagnostic accuracy (area under the receiver-operating characteristic curve, [AUC]) of 0.70 [13]. In 2009, Jeldres et al. also reported a predictive model including 5 variables (cold ischemia time [CIT], recipient age, human leukocyte antigen [HLA] mismatch, panel reactive antibody [PRA], donor age) with an AUC of 0.74 for 1219 recipients [15]. In 2014, Chapal et al. proposed a predictive score calculated using 5 parameters (CIT, donor age, recipient Body Mass Index [BMI], donor serum creatinine, anti-thymocyte globulin [ATG] induction) with an AUC of 0.73 [12]. In 2015, Zaza et al. have developed a pre-operative predictive model for DGF using 4 variables from the recipient (recipient weight, recipient previous transplant, dialysis way, duration of dialysis) with an AUC of 0.63 for 2,755 patients undergoing deceased donor kidney transplantation [16].

Even though these predictive tools performed well with good discrimination ability to predict DGF risk; however, these models were developed and validated mainly based on patients in the Western countries. The predictive accuracies of these models for Chinese population remain to be investigated. Currently, there is still no DGF predictive model specifically developed for the Chinese population. To identify an appropriate predictive model of DGF risk after deceased donor kidney transplantation for the Chinese population, this study aimed to evaluate the predictive accuracies of the aforementioned five models by external validation using the data of 713 kidney transplantation cases at our center.

\section{RESULTS}

\section{Demographics and characteristics of donors and recipients}

A total of 711 renal transplant cases using deceased donors were included for analysis. According to the status of DGF occurrence, the 711 patients were divided into DGF $(n=125)$ and non-DGF $(n=586)$ groups. The overall incidence of DGF was $17.6 \%$. The demographic and clinical characteristics of donor and recipient were summarized in Table 1. Except for age $(P<0.01)$, there was no significance in the characteristics of recipients between DGF and non-DGF groups (all $P>0.05$ ). Donors in DGF group had significantly higher WIT, CIT, terminal serum creatinine, hypertension, cardiac death than those in non-DGF group (all $P<0.05$ ). The 1-year, 3-year, 5 -year graft survival were $92.6 \%, 89.0 \%$ and $89.0 \%$ in DGF group, while were $94.6 \%, 90.2 \%$ and $90.2 \%$ in nonDGF group ( $p=0.498$ ). The 1-year, 3-year, 5-year patient survival were $96.8 \%, 94.5 \%$ and $94.5 \%$ in DGF group, while were $97.1 \%, 94.9 \%$ and $94.9 \%$ in non-DGF group $(p=0.946)$.

\section{The association between the observed DGF incidences and the calculated DGF risks}

The number of patients included for validation of the five models was shown in the Supplementary Table 2. Univariate logistic regression was used to analyze the relations between the observed DGF incidences and the calculated DGF risks. Except for Jeldres 2009 model, there were significant associations between the observed DGF incidence and the calculated DGF risks in the other four predictive models (all $P<0.01$, Table 2). Among them, Irish 2010 model had the lowest -2 log-likelihood value and the highest Nagelkerke $\mathrm{R}^{2}$ value among the five models (Table 2), suggesting that the calculated DGF risk from Irish 2010 model was most associated with the observed DGF incidence among the five models.

\section{Assessment of discrimination ability}

The discrimination ability of the five models was assessed by ROC curve using the observed DGF incidence as the standard. As shown in Figure 1, Irish 2010 model consistently had the highest area under ROC curve, $(\mathrm{AUC}=0.737,95 \%$ confidence interval $=0.684$ 0.790 ) among the five predictive models, indicating a fair predictive power of Irish 2010 model on the patients of this study. The AUC values for other four models were all small than 0.6 , suggesting a poor predictive power.

\section{Assessment of calibration}

The calibration of models was evaluated by HosmerLemeshow goodness-of-fit test. As shown in the Figure 2, the Irish 2010 model had a perfect calibration between the calculated DGF risk and the observed DGF incidence ( $p=0.887$, Figure 2A), whereas the other four models had poor calibration (both $p<0.05$, Figure $2 \mathrm{~B}-2 \mathrm{E}$ ).

\section{Subgroup analyses}

To preliminarily evaluate the predictive accuracy, the calculated DGF risks from the five models were compared between DGF and non-DGF patients in several subgroup analyses including DBD, DCD, ECD, adult donor, child donor, donor aged 5-18 and donor aged $\leq$ 5 . The results showed that in the subgroup analyses of DBD, adult donor and child donor, the calculated DGF 
Table 1: Demographic and clinical characteristics of donors and recipients at time of transplantation

\begin{tabular}{lllll}
\hline Characteristics & Total $(\boldsymbol{n}=\mathbf{7 1 1})$ & DGF $(\boldsymbol{n}=\mathbf{1 2 5})$ & non-DGF $(\boldsymbol{n}=\mathbf{5 8 6})$ & $\boldsymbol{P}$ \\
\hline Recipient & & & & \\
Age (years) & $42(32-52)$ & $38(29-48)^{*}$ & $43(33-53)$ & 0.003 \\
Weight (kg) & $60(50-65)$ & $59(50-66)$ & $60(50-65)$ & 0.388 \\
BMI (kg/m $)^{2}$ & $21.1(18.6-23.8)$ & $21.3(18.7-24.5)$ & $21.0(18.6-23.7)$ & 0.618 \\
Gender (\%male) & $467(65.7)$ & $84(67.2)$ & $383(65.4)$ & 0.411 \\
Single Organ Transplantation & $702(98.7)$ & $125(100.0)$ & $577(98.5)$ & 0.373 \\
Previous Transplants (\%) & $21(3)$ & $1(0.8)$ & $20(3.4)$ & 0.150 \\
Diabetes (\%) & $94(13.2)$ & $12(9.6)$ & $82(14.0)$ & 0.188 \\
Transfusion (\%) & $135(19)$ & $22(17.6)$ & $113(19.2)$ & 0.628 \\
Duration of Dialysis (days) & $360(120-752.5)$ & $360(118.5-752)$ & $360(120-752.75)$ & 0.558 \\
PD (\%) & $174(24.5)$ & $38(30.4)$ & $135(23.0)$ & 0.067 \\
HLA mismatch & $4(3-4)$ & $4(4-4)$ & $4(3-4)$ & 0.126 \\
ATG induction (\%) & $654(92.0)$ & $116(92.8)$ & $536(91.8)$ & 0.667 \\
Donor & & & & \\
Age (years) & $28(18-41)$ & $33(19-42)$ & $28(18-41)$ & 0.324 \\
Weight (kg) & $60(50-70)$ & $61.5(45-70)$ & $60(50-69)$ & 0.291 \\
WIT (minutes) & $0(0-7)$ & $4.5(0-15)^{*}$ & $0(0-7)$ & $<0.001$ \\
CIT (hours) & $10(6.8-15.5)$ & $11.9(8-24)^{*}$ & $9.6(6.6-14.3)$ & 0.003 \\
Terminal Scr (mg/dL) & $1.0(0.7-1.5)$ & $1.22(0.92-2.23)^{*}$ & $1.0(0.6-1.4)$ & $<0.001$ \\
Hypertension (\%) & $89(12.5)$ & $20(16)^{*}$ & $69(11.8)$ & 0.034 \\
Cardiac death (\%) & $316(44.4)$ & $85(68.0)^{*}$ & $231(39.4)$ & $<0.001$ \\
Anoxia (\%) & $43(6.0)$ & $7(5.6)$ & $36(6.1)$ & 0.817 \\
Cerebrovascular death (\%) & $134(18.8)$ & $18(14.4)$ & $116(19.8)$ & 0.161 \\
Expanded Criteria Donor1 (\%) & $39(5.5)$ & $11(8.8)$ & $28(4.8)$ & 0.070 \\
DGF (\%) & $125(17.6)$ & & & \\
\hline DGF & & & \\
\hline
\end{tabular}

DGF, delayed graft function; BMI, body mass index; PD, peritoneal dialysis; HLA, human leukocyte antigen; ATG, antithymocyte globulin; WIT, warm ischemia time; CIT, cold ischemia time. ${ }^{1}$ Defined as all donors older than 60 years of age or donor older than 50 years with any two of the following conditions: (1) history of hypertension; (2) terminal serum creatinine $>1.5 \mathrm{mg} / \mathrm{dL}$; and (3) cerebrovascular cause of brain death.

Table 2: The relationship between observed DGF incidence and calculated DGF risk analyzed by univariate logistic regression

\begin{tabular}{ccccc}
\hline Model & $\boldsymbol{P}$ value & OR $(\mathbf{9 5 \%}$ CI) & -2LL & Nagelkerke R $^{2}$ \\
\hline Irish 2010 (per 1\% increase) & $<0.001$ & $1.054(1.039-1.069)$ & 487.000 & 0.162 \\
Irish 2010 ( $\geq 20 \%$ vs $<20 \%)$ & $<0.001$ & $4.634(2.969-7.233)$ & 501.818 & 0.125 \\
Irish 2003 (per 1\% increase) & $<0.001$ & $1.041(1.028-1.053)$ & 507.422 & 0.119 \\
Chaphal 2014 (per 1\% increase) & $<0.001$ & $1.049(1.030-1.068)$ & 510.390 & 0.074 \\
Zaza 2015 (per 1\% increase) & $<0.01$ & $1.025(1.008-1.042)$ & 584.513 & 0.021 \\
Jeldres 2009 (per 1\% increase) & 0.684 & $1.005(0.980-1.031)$ & 541.239 & $<0.001$ \\
\hline
\end{tabular}

OR, odds ratio; CI, confidence interval; LL, log likelihood.

risk was significantly different between DGF and nonDGF patients in the four models (Irish 2010, Irish 2003, Chaphal 2014, Zaza 2015, all $P<0.05$, Table 3). In the subgroup analysis of donor aged $5-18$, the significance was observed in the three models (all $P<0.05$ ). There were only two and one significances in the DCD and donor aged $\leq 5$ subgroups analyses, respectively. Notably, except for ECD subgroup analysis, Irish 2010 model 
achieved significance in all subgroup analyses. However, we found that in the subgroup of donor aged $\leq 5$, there was a large gap between the observed incidence and the calculated risk of DGF by Irish 2010 model (27\% vs 9\%).

\section{Cut-off values determination- clinical usefulness}

Hence, different cut-off values (0.1-0.5) were applied to the ROC curve of Irish 2010 model, and the relevant parameters were shown in Table 4 . The optimal cut-off values were selected based on the maximum Youden index, defined as the difference between the sensitivity and 1-specificity on the ROC curve [17]. According to the maximum Youden index (36.08\%), the optimal cut-off value was 0.2 , with a sensitivity of $60.78 \%$ and a specificity of $75.3 \%$ (Table 4 ). At the cut-off of 0.1 , the model had the best sensitivity $(82.35 \%)$, negative predictive value $(93.08 \%)$ and negative likelihood ratio (0.37), while at the cut-off of 0.5 , the model had the best specificity (98.01\%), positive predictive value $(61.54 \%)$ and positive likelihood ratio (7.88, Table 4).

\section{DISCUSSION}

This is the first study evaluating the predictive models for DGF after deceased kidney transplantation for the Chinese population. In this study, we evaluated the predictive power of five available models. Univariate logistic regression showed that the calculated DGF risk from Irish 2010 model was most associated with the observed DGF incidence among the five models. Irish 2010 model had an AUC of 0.737. Hosmer-Lemeshow

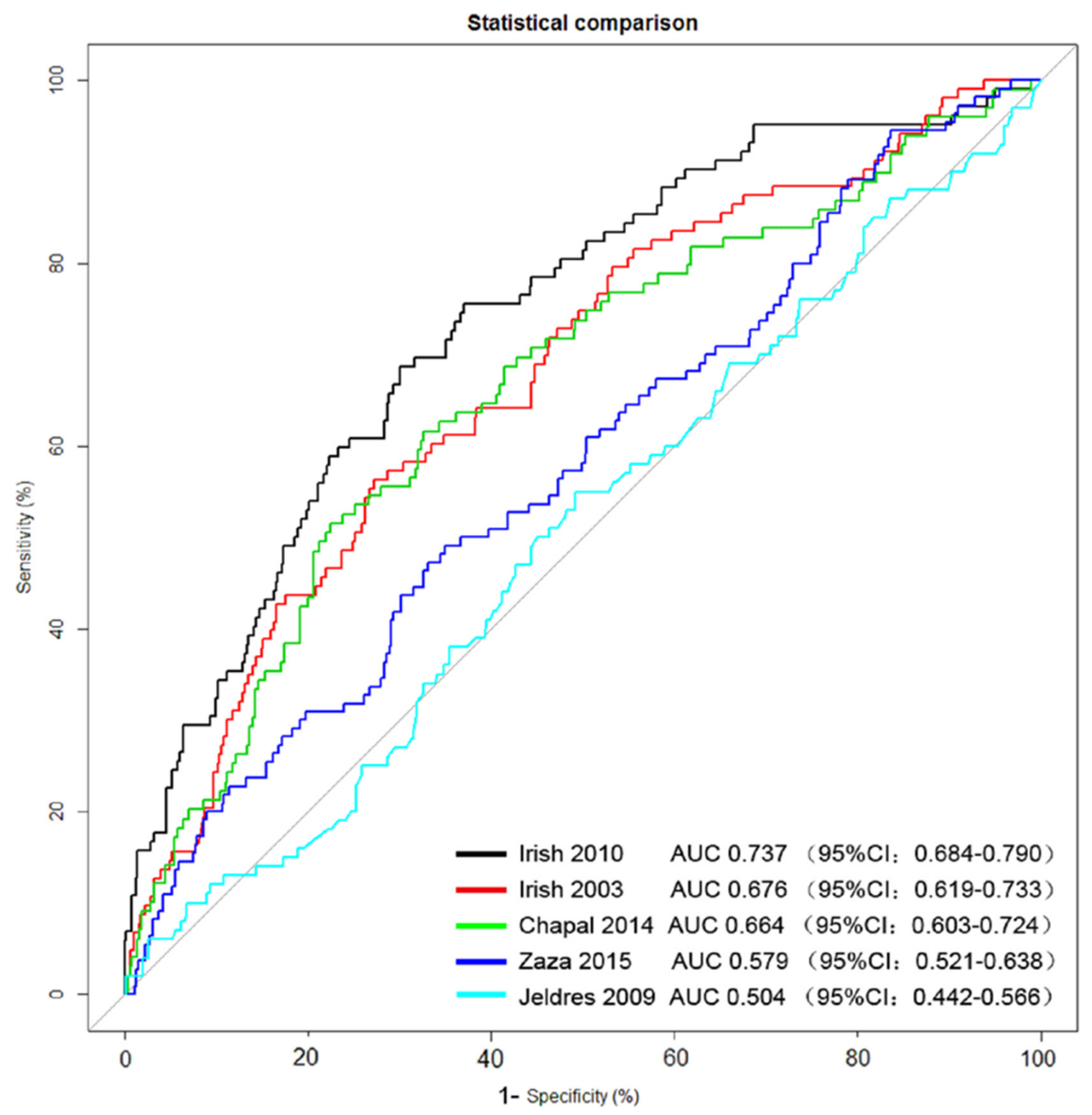

Figure 1: Evaluation of the predictive power of the five models by receiver operating characteristic (ROC) curve using the observed DGF incidence as the standard. AUC, area under the ROC curve; CI, confidence interval. 
Table 3: Comparisons of calculated DGF risks from the five models between DGF and non-DGF patients in several subgroup analyses

\begin{tabular}{|c|c|c|c|c|c|c|c|}
\hline \multirow[b]{2}{*}{ Subgroup } & \multirow{2}{*}{$\begin{array}{c}\text { Observed } \\
\text { incidence of } \\
\text { DGF }(95 \% \text { CI })\end{array}$} & \multirow[b]{2}{*}{ Model } & \multirow{2}{*}{$\begin{array}{c}\text { Total } \\
\text { included } \\
\text { cases }\end{array}$} & \multicolumn{3}{|c|}{ Calculated DGF risk (median) } & \multirow[b]{2}{*}{$P$ value } \\
\hline & & & & DGF group & $\begin{array}{l}\text { Non-DGF } \\
\text { group }\end{array}$ & All & \\
\hline \multirow{5}{*}{ DBD } & \multirow{5}{*}{$\begin{array}{l}0.10 \\
(0.07-0.13)\end{array}$} & Irish 2010 & 372 & 0.12 & 0.07 & 0.07 & $<0.001^{*}$ \\
\hline & & Irish 2003 & 372 & 0.31 & 0.22 & 0.23 & $0.001^{*}$ \\
\hline & & Chaphal 2014 & 371 & 0.34 & 0.25 & 0.27 & $<0.001^{*}$ \\
\hline & & Zaza 2015 & 380 & 0.51 & 0.44 & 0.44 & $0.03^{*}$ \\
\hline & & Jeldres 2009 & 371 & 0.08 & 0.09 & 0.09 & 0.94 \\
\hline \multirow{5}{*}{ DCD } & \multirow{5}{*}{$\begin{array}{l}0.28 \\
(0.22-0.34)\end{array}$} & Irish 2010 & 232 & 0.28 & 0.21 & 0.23 & $<0.001^{*}$ \\
\hline & & Irish 2003 & 233 & 0.52 & 0.47 & 0.48 & 0.12 \\
\hline & & Chaphal 2014 & 228 & 0.35 & 0.28 & 0.31 & $0.01^{*}$ \\
\hline & & Zaza 2015 & 275 & 0.47 & 0.47 & 0.47 & 0.28 \\
\hline & & Jeldres 2009 & 231 & 0.09 & 0.08 & 0.08 & 0.68 \\
\hline \multirow{5}{*}{ ECD } & \multirow{5}{*}{$\begin{array}{l}0.28 \\
(0.13-0.43)\end{array}$} & Irish 2010 & 39 & 0.23 & 0.19 & 0.21 & 0.12 \\
\hline & & Irish 2003 & 39 & 0.44 & 0.47 & 0.46 & 0.96 \\
\hline & & Chaphal 2014 & 39 & 0.36 & 0.35 & 0.38 & 0.68 \\
\hline & & Zaza 2015 & 39 & 0.45 & 0.49 & 0.48 & 0.43 \\
\hline & & Jeldres 2009 & 39 & 0.14 & 0.18 & 0.18 & 0.40 \\
\hline \multirow{5}{*}{$\begin{array}{l}\text { Adult donor } \\
(\geq 18 \text { year old })\end{array}$} & \multirow{5}{*}{$\begin{array}{l}0.18 \\
(0.15-0.22)\end{array}$} & Irish 2010 & 481 & 0.24 & 0.11 & 0.12 & $<0.001^{*}$ \\
\hline & & Irish 2003 & 482 & 0.47 & 0.30 & 0.33 & $<0.001^{*}$ \\
\hline & & Chaphal 2014 & 479 & 0.35 & 0.27 & 0.30 & $<0.001^{*}$ \\
\hline & & Zaza 2015 & 501 & 0.46 & 0.44 & 0.44 & $0.02^{*}$ \\
\hline & & Jeldres 2009 & 480 & 0.10 & 0.10 & 0.10 & 0.80 \\
\hline \multirow{5}{*}{$\begin{array}{l}\text { Child donor }(<18 \\
\text { year old })\end{array}$} & \multirow{5}{*}{$\begin{array}{l}0.14 \\
(0.08-0.20)\end{array}$} & Irish 2010 & 123 & 0.22 & 0.08 & 0.09 & $<0.001^{*}$ \\
\hline & & Irish 2003 & 123 & 0.43 & 0.23 & 0.25 & $0.04^{*}$ \\
\hline & & Chaphal 2014 & 120 & 0.28 & 0.19 & 0.23 & $0.04^{*}$ \\
\hline & & Zaza 2015 & 125 & 0.52 & 0.47 & 0.48 & $0.04^{*}$ \\
\hline & & Jeldres 2009 & 122 & 0.03 & 0.04 & 0.04 & 0.12 \\
\hline \multirow{5}{*}{ Donor aged 5-18 } & \multirow{5}{*}{$\begin{array}{l}0.10 \\
(0.04-0.16)\end{array}$} & Irish 2010 & 100 & 0.26 & 0.08 & 0.09 & $0.01^{*}$ \\
\hline & & Irish 2003 & 100 & 0.45 & 0.27 & 0.28 & $0.03^{*}$ \\
\hline & & Chaphal 2014 & 99 & 0.33 & 0.22 & 0.26 & $0.02^{*}$ \\
\hline & & Zaza 2015 & 101 & 0.49 & 0.43 & 0.43 & 0.33 \\
\hline & & Jeldres 2009 & 99 & 0.04 & 0.04 & 0.04 & 0.56 \\
\hline \multirow{5}{*}{ Donor aged $\leq 5$} & \multirow{5}{*}{$\begin{array}{l}0.27 \\
(0.12-0.39)\end{array}$} & Irish 2010 & 40 & 0.21 & 0.06 & 0.09 & $0.01^{*}$ \\
\hline & & Irish 2003 & 40 & 0.32 & 0.17 & 0.18 & 0.20 \\
\hline & & Chaphal 2014 & 38 & 0.21 & 0.17 & 0.17 & 0.53 \\
\hline & & Zaza 2015 & 41 & 0.53 & 0.53 & 0.53 & 0.34 \\
\hline & & Jeldres 2009 & 40 & 0.02 & 0.02 & 0.02 & 0.86 \\
\hline
\end{tabular}

DGF, delayed graft function; CI, confidence interval; DBD, donation after brain death; DCD, donation after cardiac death; ECD, expanded criteria donors. "Significance in the calculated DGF risk between DGF and non-DGF patient

goodness-of-fit test showed that the Irish 2010 model had a perfect calibration between the calculated DGF risk and the observed DGF incidence $(p=0.887)$. The calculated DGF risk from Irish 2010 model was significantly different between DGF and non-DGF patients in the subgroup analyses of DBD, DCD, adult donor, child donor, donor aged 5-18 and donor aged $\leq 5$, but not ECD. Taken together, these data suggested that 
Table 4: ROC curve analysis of Irish 2010 model with different cut-offs

\begin{tabular}{cccccccc}
\hline Cut-off & Sensitivity & Specificity & $\begin{array}{c}\text { Positive } \\
\text { predictive } \\
\text { value }\end{array}$ & $\begin{array}{c}\text { Negative } \\
\text { predictive } \\
\text { value }\end{array}$ & $\begin{array}{c}\text { Positive } \\
\text { likelihood } \\
\text { ratio }\end{array}$ & $\begin{array}{c}\text { Negative } \\
\text { likelihood } \\
\text { ratio }\end{array}$ & $\begin{array}{c}\text { Youden } \\
\text { index }\end{array}$ \\
\hline 0.1 & $82.35 \%$ & $48.21 \%$ & $24.42 \%$ & $93.08 \%$ & 1.59 & 0.37 & $30.56 \%$ \\
0.2 & $60.78 \%$ & $75.30 \%$ & $33.33 \%$ & $90.43 \%$ & 2.46 & 0.52 & $36.08 \%$ \\
0.3 & $36.27 \%$ & $88.45 \%$ & $38.95 \%$ & $87.23 \%$ & 3.14 & 0.72 & $24.72 \%$ \\
0.4 & $22.55 \%$ & $95.42 \%$ & $50.00 \%$ & $85.84 \%$ & 4.92 & 0.81 & $17.97 \%$ \\
0.5 & $15.69 \%$ & $98.01 \%$ & $61.54 \%$ & $85.12 \%$ & 7.87 & 0.86 & $13.69 \%$ \\
\hline
\end{tabular}
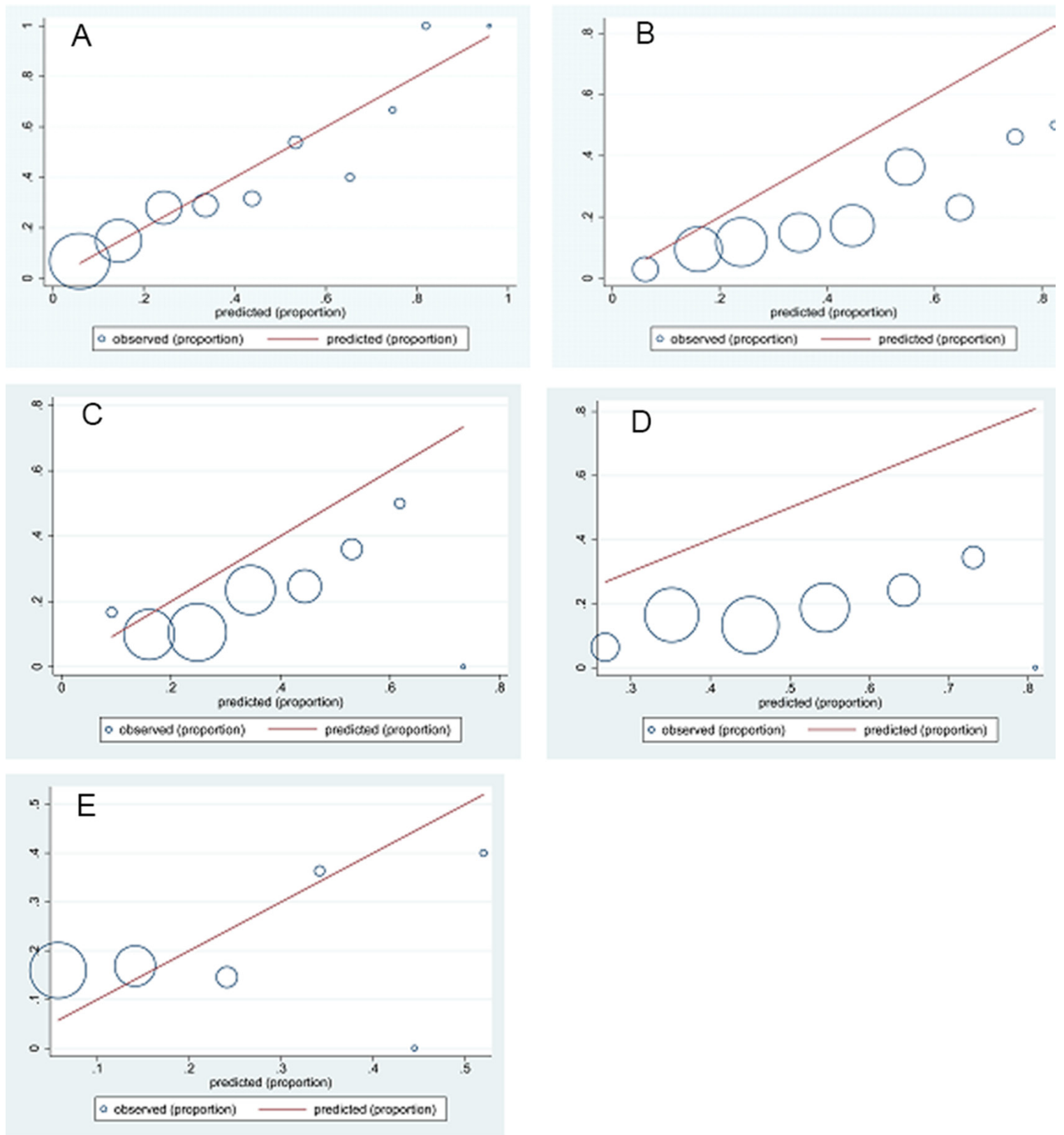

Figure 2: Evaluation of the model calibration by the Hosmer-Lemeshow goodness-of-fit test for Irish 2010 model (A), Irish 2003 model (B), Chaphal 2014 model (C), Zaza 2015 model (D) and Jeldres 2009 model (E). The observed and predicted delayed graft function (DGF) probabilities were computed according to 10 delayed graft function score (DGF) with intervals at 0.1 . The goodness of the fit for Irish 2010 model (A) cannot be rejected ( $P=0.887$, Hosmer-Lemeshow statistic). Both the goodness of the fit for Irish 2003 model (B), Chaphal 2014 (C), Zaza 2015 (D) and Jeldres 2009 (E) were rejected $(P<0.05$, Hosmer-Lemeshow statistic). 
Irish 2010 model has the best predictive power among the five models.

In our population, there were significant differences in the recipient age, WIT, CIT, donor terminal serum creatinine, donor history of hypertension and donation after cardiac death between DGF and non-DGF group, most of which was consistent with findings of previous reports $[8,18]$. The recipient age was younger in the DGF group as compared with the non-DGF group (38 [29-48] vs. 43 [33-53], $P=0.003)$. The difference in recipient age between DGF group and non-DGF group in our cohort may be attributed to the integrative factors in clinical practice, including organ allocation, surgical decision and patient's willingness. However, among four out of the five models (Irish 2010, Irish 2003, Chaphal 2014, Zaza 2015, all of them with a large sample size) validated in this study, recipient age is not a predictor of DGF, hence there is no age bias in the external validation of these four models. In Jeldres 2009 model $(N=532)$, age $>43$ years increases the risk of DGF. In our validation cohort, the proportion of recipients aged $>43$ was lower in the DGF group than in the non-DGF group $(38.4 \% v s .51 .5 \%, p=0.008)$. The lower proportion of recipients aged $>43$ in our validation cohort did underestimate the calculated risk in DGF group. Nevertheless, the absolute difference in the proportion of recipients aged $>43$ between DGF group and non-DGF group in our cohort was relatively small $(13.1 \%)$. In addition, among all the included parameters in the Jeldres 2009 model, the recipient age has the least effect on the DGF risk [15]. Therefore, we believed that the age bias in our validation cohort would not alter the conclusion that the discrimination and calibration ability of Jeldres 2009 model was poor for the Chinese population.

The Irish 2010 model has good discrimination and perfect calibration for the Chinese population. Several reasons may contribute to its good predictive power. First, the Irish 2010 model was refined from the Irish 2003 model and made improvements in more risk factors (includes 17 conventional predictors) such as WIT, and more specific inclusion criteria. In addition, Irish 2010 model was developed using the data from the United Network for Organ Sharing (UNOS), which is the largest database among the five models and includes different races, both reduce the selection bias in the logistic regression model. Furthermore, this model has been externally validated using the UNOS 2007 validation dataset as well as Spanish and Belgian population dataset $[13,19,20]$.

It is worth mentioning there are some differences in defining and detecting the clinical variables when we externally validated the Irish 2010 model using the data from our population. For instance, PRA variable in our dataset was recorded as "positive or negative PRA" of PRA rather than "percentage of PRA". Since the mean of PRA in Irish 2010 model was approximately $20 \%$, therefore the variables of positive and negative PRA in our dataset were coded as $20 \%$ and $0 \%$, respectively. However, the maximum difference between positive and negative PRA is merely 2 score according to the nomogram of Irish 2010 model, which has little influence on the applicability of the models in China.

To provide references for medical decision making, we compared different cut-offs for the calculated DGF risk from the Irish 2010 model. The first selection method for an appropriate cut-off value is based on the maximum Youden index. At the optimal cut-off of 0.2, the model has the best discrimination. The second method is based on the positive and negative likelihood ratios. At the cutoff of 0.1 , the model has the best sensitivity and negative likelihood ratio, while at the cut-off of 0.5 , the model has the best specificity and positive likelihood ratio. We believe that these cut-offs would be better because they are less associated with the baseline level of DGF incidence and of more practical value in the clinic. For patients with a calculated DGF risk less than the lower cut-off (i.e. 0.1), DGF could be excluded. For patients with a calculated DGF risk higher than the upper cut-off (i.e. 0.5), appropriate preventive managements or pretreatments should be conducted to prevent the occurrence of DGF and avoid deleterious consequences on long-term graft outcome.

In this study, the overall incidence of DGF was $17.6 \%$, and the DGF rates for DBD and DCD cases were $10 \%$ and $28 \%$, respectively. The incidences of DGF in the current study are in line with several recent reports of Chinese population from the other large transplant centers [21-23] and the donor and recipient characteristics are similar between these large transplant centers. These evidences indicated the study population in our center was representative of the national population. The incidence of DGF is lower in our study than in the USA (e.g. Irish et al.'s 2010 study, DBD: 25.5\%; DCD: $48.2 \%$ [13]). One explanation is that the donors in our study were younger; the cause of death was mainly car accident, and the proportion of cerebrovascular accident death was low (18.8\%). While in the USA, the proportion of cerebrovascular accident death is usually higher than $40 \%$, and exhibits a gradually rising trend [24]. In addition, the donor in our study had a lower incidence of hypertension than those in Irish et al.'s 2010 study (12.5\% vs $22.4 \%$ ). Furthermore, the warm ischemic time and cold ischemic time in our study were both shorter than those in Irish et al.'s 2010 study (WIT $=39.3 \pm 17.6 \mathrm{~min}$;

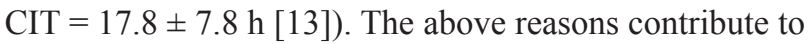
a low risk of DGF in our study cohort.

Although the Irish 2010 model has the best performance for our population among the five models, it still has several limitations. The Irish 2010 model has an AUC of 0.737 for our population, suggesting there is still room for improvement. To improve the discrimination performance, additional risk factors may be considered to be included in the model. For example, 
ATG induction $[8,25]$, recipient's residual renal function and perioperative saline loading have been reported be associated with DGF [26], which could be considered to update the Irish 2010 model for the Chinese population. In the several subgroup analyses of this study, the calculated DGF risk between DGF and non-DGF patients reached significance in all subgroup analyses except for ECD, which may be attributed to the small sample size of ECD subgroup analysis $(n=39)$. In the subgroup of donor aged $\leq 5$, the observed DGF incidence is up to $27 \%$, whereas the calculated DGF risk by Irish 2010 model is only $9 \%$. One possible explanation for this difference may be that among our subgroup of donor aged $\leq 5$, there were about $40 \%$ (15 cases) of donors weighing $\leq 10 \mathrm{~kg}$, and their high incidence of postoperative vascular and ureteral complications would lead to a high risk of DGF [27]. While in the Irish 2010 model, the proportion of donor weighed $\leq 10 \mathrm{~kg}$ is extremely small $(0.34 \%$ of all deceased donor) [28], therefore this issue could not be considered in the model. However, we cannot conduct further subgroup analysis within the subgroup of donor aged $\leq 5$ due to its small size $(n=40)$. Therefore a study with a large sample size is necessary to validate this issue and to figure out an appropriate predictive model for this subgroup of recipients. It should also be noted that we used the data from a single center in China, and the sample size is still relatively small. It has been suggested that external validation of a prognostic model requires a minimum of 100 events $[29,30]$, which is much smaller than that for model development (rule of thumb: 5-20 events per variable) [31]. In our validation cohort, 125 events (DGF) were included, thus it was sufficient for unbiased estimation of performance measures of the five models. However, a multicenter study with large sample size should be performed to validate our findings. All these limitations should be addressed in the following study.

\section{MATERIALS AND METHODS}

\section{Study population}

To evaluate the predictive power of the five models (Irish 2010 [13], Irish 2003 [14], Chaphal 2014 [12], Zaza 2015 [16], Jeldres 2009 [15]) for the DGF risk after deceased donor kidney transplantation for Chinese population, the medical records of 713 patients receiving solitary renal transplantation using deceased donors from organ donation after brain death (DBD) and circulatory death (DCD) [32] in the First Affiliated Hospital, Sun Yatsen University between February 2007 and August 2016 were retrospectively reviewed. No organs from executed prisoners were used. Two patients without the record of DGF status were excluded from the analysis. In addition, the patients were screened for the validation cohort of the five models according to their own inclusion criteria (Supplementary Table 1, Supplementary Figure 1). In the analysis for the Irish 2010 model and Irish 2003 model, the recipients aging $<16$ years were excluded, while the recipients aging $<18$ years were excluded in the other models. This study was approved by the institutional review board of the First Affiliated Hospital of Sun Yatsen University.

\section{Data collection}

Data including demographic and pre-/posttransplant-related clinical characteristics of donors and recipients, follow-up events and outcomes were collected. DGF was defined as the need for dialysis within the first week post-transplant [2]. The five predictive models were presented in different formats including regression formula, nomogram, web-based calculator and scoring system (Supplementary Table 2). The DGF risk was mainly calculated from the regression formula in the model. If the regression formula was not available in the model, the DGF risk was calculated for each recipient using nomogram, web-based calculator or scoring system. The higher the calculated DGF risk is, the more likelihood that DGF develops in the patient. The variables included for analysis of the five predictive models were listed in Supplementary Table 2. We had complete data for most of the variables; while there was some missing data (< 5-10\%, except for PRA) needed imputation. They were missing at random. Calculated PRA (cPRA) was not available in China. Therefore, negative and positive PRA in our dataset were coded as $0 \%$ and $20 \%$ (median of cPRA in Irish 2010 model). Missing duration of dialysis was recorded as 360 days (median of available data). Missing HLA mismatches was recorded as 4 (median of available mismatch number). Missing donor terminal serum creatinine was considered normal and estimated with age and sex according to the modification of diet in renal disease (MDRD) equation ( $>16$ years old) [33] or Schwartz equation $(\leq 16$ years old) (Normal GFR $=$ $90 \mathrm{ml} / \mathrm{min} / 1.73 \mathrm{~m}^{2}$ ) [34]. Missing donor weight was estimated with age and sex according to data from General Administration of Sport [35]. For categorical data, missing or unknown were grouped into the "no" or "absence" category.

\section{Statistical analysis}

Continuous data with normal distribution was presented as the mean \pm standard deviation (SD), while continuous data without normal distribution was expressed as median (lower quartile-higher quartile). Categorical data was reported as counts and percentages. KaplanMeier method was used for survival analysis and the logrank test was used to compare two survival curves. The relations between the observed incidence of DGF and the calculated DGF risk were analyzed using univariate logistic regression. Nagelkerke $\mathrm{R}^{2}$ and -2Log Likelihood 
were used for the evaluation of the regression model performance. The predictive accuracy of the five models on our data was assessed using the concordance index (AUC), which estimates the probability of concordance between predicted and observed responses. The cutoff value was determined based on the Youden index as well as a positive/negative likelihood ratio. The HosmerLemeshow "goodness-of-fit" test was taken for model calibration [36]. The calculated DGF risks from the five models were compared between DGF and non-DGF patients using Mann-Whitney U test. Two-group data comparison, ROC curve and logistic regression model were analyzed using IBM SPSS Statistics version 22.0 (IBM Corporation, New York, USA). Hosmer-Lemeshow "goodness-of-fit" test was performed with Stata MP version 14.0 (STATA Corporation, Texas, USA). Model calibration was performed using ' $R$ ', a free software environment for statistical computing and graphics (www.r-project.org).

\section{CONCLUSIONS}

In summary, our study shows that Irish 2010 model has good predictive power for DGF risk after deceased donor kidney transplantation in the Chinese population, which may be helpful in optimizing organ utilization decision and guiding preventive and therapeutic strategies.

\section{Abbreviations}

DGF: Delayed graft function; ECD: expanded criteria donors; DCD: donation after cardiac death; CIT: cold ischemia time; HLA: human leukocyte antigen; PRA: panel reactive antibody; DBD: donation after brain death; DCD: donation after circulatory death; MDRD: modification of diet in renal disease; AUC: assessed using the concordance index; UNOS: United Network for Organ Sharing

\section{ACKNOWLEDGMENTS AND FUNDING}

We extended our great gratitude to Professor Xianchang $\mathrm{Li}$, the director of Immunobiology and Transplant Science Center, Houston Methodist, Weill Cornell Medical College for his expert advice to our manuscript. This study was supported by the National Natural Science Foundation of China (No. 81670680, 81270836, 81300623), Science and Technology Planning Project of Guangdong Province, China (2015B020226002, 2014B020212006, 2013B021800292, 2014A020212719, 2014A020212142, 2013B051000020, 2010B031600236), the Natural Science Foundation of Guangdong Province (2015A030313135, 2014A030313022), Science and Technology Program of Guangzhou, China (2014Y200114, 201400000003-3), and the Guangdong Provincial Key Laboratory on Organ Donation and Transplant Immunology (2013A 061401007).

\section{CONFLICTS OF INTEREST}

The authors of this manuscript have no conflicts of interest to disclose.

\section{REFERENCES}

1. Wekerle T, Segev D, Lechler R, Oberbauer R. Strategies for long-term preservation of kidney graft function. Lancet. 2017; 389:2152-62.

2. Yarlagadda SG, Coca SG, Garg AX, Doshi M, Poggio E, Marcus RJ, Parikh CR. Marked variation in the definition and diagnosis of delayed graft function: A systematic review. Nephrol Dial Transplant. 2008; 23:2995-3003.

3. Siedlecki A, Irish W, Brennan DC. Delayed graft function in the kidney transplant. J Transplant. 2011; 11:2279-96.

4. Sharif A, Borrows R. Delayed Graft Function After Kidney Transplantation: The Clinical Perspective. Am J Kidney Dis. 2013; 62:150-8.

5. Heilman RL, Mathur A, Smith ML, Kaplan B, Reddy KS. Increasing the Use of Kidneys From Unconventional and High-Risk Deceased Donors. Am J Transplant. 2016; 16:3086-92.

6. Summers DM, Watson CJ, Pettigrew GJ, Johnson RJ, Collett D, Neuberger JM, Bradley JA. Kidney donation after circulatory death (DCD): state of the art. Kidney Int. 2015; 88:241-9.

7. Chaumont M, Racapé J, Broeders N, El Mountahi F, Massart A, Baudoux T, Hougardy JM, Mikhalsky D, Hamade A, Le Moine A, Abramowicz D, Vereerstraeten P. Delayed Graft Function in Kidney Transplants: Time Evolution, Role of Acute Rejection, Risk Factors, and Impact on Patient and Graft Outcome. J Transplant. 2015; 2015:1-9.

8. Nashan B, Abbud-Filho M, Citterio F. Prediction, prevention, and management of delayed graft function: where are we now? Clin Transplant. 2016; 30:1198-208.

9. Yacoub R, Nadkarni GN, Cravedi P, He JC, Delaney VB, Kent R, Chauhan KN, Coca SG, Florman SS, Heeger PS, Murphy B, Menon MC. Analysis of OPTN/UNOS registry suggests the number of HLA matches and not mismatches is a stronger independent predictor of kidney transplant survival. Kidney Int. 2017 Sep 29. [Epub ahead of print].

10. Perasaari JP, Kyllonen LE, Salmela KT, Merenmies JM. Pre-transplant donor-specific anti-human leukocyte antigen antibodies are associated with high risk of delayed graft function after renal transplantation. Nephrol Dial Transplant. 2016; 31:672-8.

11. Redfield RR, Scalea JR, Zens TJ, Muth B, Kaufman DB, Djamali A, Astor BC, Mohamed M. Predictors and outcomes of delayed graft function after living-donor kidney transplantation. Transpl Int. 2016; 29:81-7.

12. Chapal M, Le Borgne F, Legendre C, Kreis H, Mourad G, Garrigue V, Morelon E, Buron F, Rostaing L, Kamar N, Kessler M, Ladrière M, Soulillou JP, et al. A 
useful scoring system for the prediction and management of delayed graft function following kidney transplantation from cadaveric donors. Kidney Int. 2014; 86:1130-9.

13. Irish WD, Ilsley JN, Schnitzler MA, Feng S, Brennan DC. A risk prediction model for delayed graft function in the current era of deceased donor renal transplantation. Am J Transplant. 2010; 10:2279-86.

14. Irish WD. Nomogram for Predicting the Likelihood of Delayed Graft Function in Adult Cadaveric Renal Transplant Recipients. J Am Soc Nephrol. 2003; 14:2967-74.

15. Jeldres C, Cardinal H, Duclos A, Shariat SF, Suardi N, Capitanio U, Hébert MJ, Karakiewicz PI. Prediction of delayed graft function after renal transplantation. Can Urol Assoc J. 2009; 3:377-82.

16. Zaza G, Ferraro PM, Tessari G, Sandrini S, Scolari MP, Capelli I, Minetti E, Gesualdo L, Girolomoni G, Gambaro G, Lupo A, Boschiero L. Predictive model for delayed graft function based on easily available pre-renal transplant variables. Intern Emerg Med. 2015; 10:135-41.

17. Fluss R, Faraggi D, Reiser B. Estimation of the Youden Index and its associated cutoff point. Biom J. 2005; 47:458-72.

18. Salazar Meira F, Zemiacki J, Figueiredo AE, Viliano Kroth L, Saute Kochhann D, d'Avila DO, Traesel M, Saitovitch D, Poli-de-Figueiredo CE. Factors Associated With Delayed Graft Function and Their Influence on Outcomes of Kidney Transplantation. Transplant Proc. 2016; 48:2267-71.

19. Rodrigo E, Miñambres E, Ruiz JC, Ballesteros A, Piñera C, Quintanar J, Fernández-Fresnedo G, Palomar R, GómezAlamillo C, Arias M. Prediction of delayed graft function by means of a novel web-based calculator: a single-center experience. Am J Transplant. 2012; 12:240-4.

20. Decruyenaere A, Decruyenaere P, Peeters P, Vermassen F. Validation in a Single-Center Cohort of Existing Predictive Models for Delayed Graft Function After Kidney Transplantation. Ann Transplant. 2015; 20:544-52.

21. Xue W, Tian P, Xiang H, Ding X, Pan X, Yan H, Hou J, Feng X, Liu L, Ding C, Tian X, Li Y, Zheng J. Outcomes for primary kidney transplantation from donation after Citizens' death in China: a single center experience of 367 cases. BMC Health Serv Res. 2017; 17:250.

22. Zhu L, Fu C, Lin K, Wang Z, Guo H, Chen S, Lin Z, Chen Z, Chen G. Patterns of Early Rejection in Renal Retransplantation: A Single-Center Experience. J Immunol Res. 2016; 2016:1-7.

23. Xiaoming P, Xiang H, LinJuan L, Chenguang D, Ren L. Preliminary results of transplantation with kidneys donated after cardiac death: a path of hope for organ transplantation in China. Nephrol Dial Transplant. 2015; 30:1590-6.

24. Nathan HM, Conrad SL, Held PJ, McCullough KP, Pietroski RE, Siminoff LA, Ojo AO. Organ donation in the United States. Am J Transplant. 2003; 3:29-40.
25. Bronzatto EJ, da Silva Quadros KR, Santos RL, Alves-Filho G, Mazzali M. Delayed Graft Function in Renal Transplant Recipients: Risk Factors and Impact on 1-Year Graft Function: A Single Center Analysis. Transplant Proc. 2009; 41:849-51.

26. Ounissi M, Cherif M, Abdallah TB, Bacha M, Hedri H, Abderrahim E, Goucha R, Kheder A, Slama RB, Derouiche A, Chebil M, Bardi R, Sfar I, et al. Risk factors and consequences of delayed graft function. Saudi J Kidney Dis Transpl. 2013; 24:243-6.

27. Kayler LK, Magliocca J, Kim RD, Howard R, Schold JD. Single kidney transplantation from young pediatric donors in the United States. Am J Transplant. 2009; 9:2745-51.

28. View data reports. Organ Procurement and Transplantation Network. https://optn.transplant.hrsa.gov/data/view-datareports/build-advanced/.

29. Collins GS, Ogundimu EO, Altman DG. Sample size considerations for the external validation of a multivariable prognostic model: a resampling study. Stat Med. 2016; 35:214-26.

30. Vergouwe Y, Steyerberg EW, Eijkemans MJ, Habbema JD. Substantial effective sample sizes were required for external validation studies of predictive logistic regression models. J Clin Epidemiol. 2005, 58:475-83.

31. Concato J, Peduzzi P, Holford T, Feinstein A. Importance of events per independent variable in proportional hazards regression analysis: I. Background, goals and general strategy. J Clin Epidemiol. 1995; 48:1495-501.

32. Huang J, Wang H, Fan ST, Zhao B, Zhang Z, Hao L, Huo F, Liu Y. The national program for deceased organ donation in China. Transplantation. 2013; 96:5-9.

33. Levey AS, Coresh J, Greene T, Stevens LA, Zhang YL, Hendriksen S, Kusek JW, Van Lente F; Chronic Kidney Disease Epidemiology Collaboration. Using standardized serum creatinine values in the modification of diet in renal disease study equation for estimating glomerular filtration rate. Ann Intern Med. 2006; 145:247-54.

34. Schwartz GJ, Muñoz A, Schneider MF, Mak RH, Kaskel F, Warady BA, Traesel M, Saitovitch D, Poli-de-Figueiredo CE. New Equations to Estimate GFR in Children with CKD. J Am Soc Nephrol. 2009; 20:629-37.

35. General Administration of Sport of China. 2014 National Fitness Monitoring Bulletin. http://www.sport.gov.cn/n16/ n1077/n1422/7331093.html.

36. Hosmer DW, Hosmer $\mathrm{T}$, Le Cessie S, Lemeshow S. A comparison of goodness-of-fit tests for the logistic regression model. Stat Med. 1997; 16:965-80. 